\title{
Ultrafast Fluorescence Dynamics in Flurbiprofen-Amino Acid Dyads and in the Supramolecular Drug/Protein Complex
}

\author{
Ignacio Vayá*a,c, M. Consuelo Jiméneza , Miguel A. Miranda ${ }^{a}$, Aninda Chatterjee ${ }^{b}$, and \\ Thomas Gustavsson*b
}

\begin{abstract}
The interaction dynamics between the drug flurbiprofen (FBP) and human serum albumin (HSA) has been investigated by time-resolved fluorescence spectroscopy, combining femtosecond fluorescence upconversion and picosecond time-correlated single photon counting. In order to obtain additional information on the drug/ protein interaction, several covalently linked model dyads, composed of FBP and tryptophan or tyrosine, were also studied. For all systems, the main feature was a remarkable dynamic FBP fluorescence quenching, more prominent in the dyads than in the protein complex. All systems also displayed a clear stereoselectivity depending on the $(S)$ - or $(R)$-form of FBP, that was strongly influenced by the conformational arrangement of the investigated chromophores.
\end{abstract}

Keywords: Drug/protein binding · Fluorescence upconversion · Flurbiprofen · Human serum albumin · Timecorrelated single photon counting $\cdot$ Time-resolved fluorescence $\cdot$ Tryptophan $\cdot$ Tyrosine

\section{Introduction}

The binding of drugs to plasma proteins constitutes an active research field because of its importance in many biological processes such as modulation of drug solubility in plasma, toxicity and in vivo half-life. ${ }^{[1]}$ The strength of binding, controlled by electrostatic, hydrogenbond and van der Waals interactions, is determinant not only for drug action but also for its transport and disposition. ${ }^{[2]}$ The extent of binding depends on a number of factors such as $\mathrm{pH}$ of the medium, nature of the protein, hydrophilicity and concentration of the drug and its affinity to the protein. Usually this process is reversible and controls the therapeutic action of the drug; ${ }^{[3]}$ as only unbound drugs are pharmacologically active, a high protein binding affinity results in greater

${ }^{\star}$ Correspondence: Dr. I. Vayáa,c, Prof. T. Gustavsson ${ }^{\mathrm{b}}$ E-mail: i.vaya-perez@uea.ac.uk, thomas.gustavsson@ cea.fr

aDepartamento de Química/Instituto de Tecnología Química UPV-CSIC

Universitat Politècnica de València

Camino de Vera s/n, 46022 Valencia, Spain 'LIDYL, Laboratoire Interactions, Dynamiques et Lasers

CEA, CNRS, Université Paris-Saclay, CEA Saclay 91191 Gif-sur-Yvette, France

'School of Chemistry, University of East Anglia

Norwich Research Park

NR4 7TJ, Norwich, United Kingdom solubility and increased half-life, whereas a low binding affinity is normally associated to limited ability to reach the site of action.

Common blood proteins that interact with drugs are human serum albumin (HSA), glycoproteins, lipoproteins and globulins. Herein, we will focus on HSA, which is the most abundant transport protein in plasma; its main physiological function is to carry endogeneous and exogeneous agents (i.e. drugs, metabolites, fatty acids, etc.) through the blood stream. ${ }^{[2,4]}$ Drug binding to HSA normally occurs in the so-called site I and site II binding sites, following Sudlow's classification. ${ }^{[5]}$ Both sites are composed of a number of amino acid residues, however only some of them play a key role in the binding process; thus, the only tryptophan (Trp) moiety W214 of HSA is located closer to site I, while site II is mainly composed of tyrosine (Tyr), histidine (His) and arginine (Arg). ${ }^{[1 a]}$ Due to their fluorescence properties, Trp and Tyr have become universal fluorescent markers (see below) for protein studies. ${ }^{[6]}$

In view of the importance of the drug/HSA binding process, a number of techniques (HPLC, ultrafiltration, calorimetry, circular dichroism, capillary electrophoresis, NMR, etc.) have been used to investigate this issue. [7] In this context, optical spectroscopies (UV/VIS/ IR absorption, fluorescence), both steadystate and time-resolved, have proven to be particularly useful.

By observing changes of the shape and the intensity of the drug's steadystate absorption spectrum in solution and in the presence of HSA, information can be obtained about ground state complex formation. ${ }^{[8]}$ Alternatively, by analysing the changes of the HSA UV absorption spectrum upon addition of a drug, information regarding the alterations of the microenvironment around the protein chromophores (Trp, Tyr, Phe) due to the complex formation can be obtained. ${ }^{[9]}$ In parallel, steady-state fluorescence spectroscopy has become a widely used technique to probe drug/protein interactions. Fluorescence quenching measurements by titration can bring valuable information about the drug/protein complex formation. The shape and the intensity of the drug's and or the protein's fluorescence spectrum are very sensitive indicators of fundamental processes such as energy and electron transfer, which can be directly related to the drug/protein binding. ${ }^{[10]}$ We recall here that the HSA fluorescence is completely dominated by its single tryptophan W214,[10] emitting in the UV spectral region. For UV emitting drugs this may lead to a strong spectral overlap, producing complex fluorescence spectra.[11]

Time-resolved spectroscopic measurements add another dimension to the study of drug/protein interactions, providing rich dynamic information. Nanosecond laser flash photolysis has proven to be extremely useful in the study of drug-binding to HSA.[12] Time-resolved fluorescence spectroscopy has been widely used to characterise the singlet excited states dynamics in the complex formation of the drug with HSA, giving key information about the strength of the interaction, conformational restrictions within the binding cavities, 
stereoselectivity in the binding process, etc. ${ }^{[12 \mathrm{~h}, 12 \mathrm{k}, 13]}$ Precise investigations need a time-resolution that is compatible with the rates of the processes involved, i.e. on the sub-nanosecond or picosecond timescale. By analysing the fluorescence decay time, one can distinguish between static and dynamical quenching processes, and the fluorescence anisotropy provides a unique means to detect the drug's attachment to HSA. Several picosecond fluorescence studies of drug/HSA complexes using timecorrelated single photon counting (TCSPC) have been reported, ${ }^{[13 b, 13 d, 14]}$ revealing detailed information on the drug/HSA interactions. An even more detailed picture has been provided using the femtosecond timeresolution offered by the fluorescence upconversion (FU) technique. ${ }^{[12 \mathrm{k}, 13 \mathrm{a}, 13 \mathrm{e}, 15]}$ The unprecedented time-resolution of FU has made it possible to distinguish between different binding sites by a dynamical decomposition of the emission spectrum into different spectro-temporal components.

Throughout the rest of this review we will focus on the drug molecule FBP (Fig. 1). FBP belongs to the class of nonsteroidal anti-inflammatory drugs (NSAIDs) which are therapeutic agents widely prescribed for the treatment of different diseases including headache, migraine, osteoarthritis, rheumatoid arthritis, fever, etc. ${ }^{[16]}$ Other drugs belong to this family are, for example, ibuprofen and naproxen. In general, although these drugs are prescribed as racemic mixtures, the anti-inflammatory activity is mainly attributed to the $(S)$-enantiomer. ${ }^{[17]}$

The binding of FBP to HSA has been well-characterised by different spectroscopic techniques. ${ }^{[7 \mathrm{~b}, 12 \mathrm{~d}, 12 \mathrm{j}, 12 \mathrm{k}, 18]}$ It has been reported that FBP binds preferentially to HSA at site II, but other low-affinity binding sites are possible. ${ }^{[19]}$

Molecular modelling, and in particular molecular docking simulations, provides an additional and very powerful tool to examine the drug/protein binding in more detail. The drug/protein interaction may involve hydrogen bonding, electrostatic interactions, hydrophobic interactions and long-range van der Waals interactions. An increasing number of docking simulations have been reported on various drug/HSA systems, informing on the precise docking sites the binding modes and the binding energies. ${ }^{[9,11,20]}$ To our knowledge, no detailed simulation study of FBP/HSA binding has been reported to this date. However, a precise modelling is outside the scope of the present work, but in order to illustrate the FBP/HSA binding a possible docking situation is illustrated in Fig. 2. We underline that this is only an example; several other docking sites are also possible.

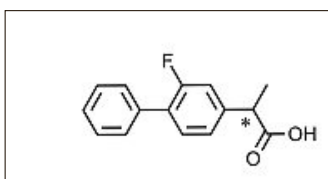

$(S)$ - or $(R)$-FBP

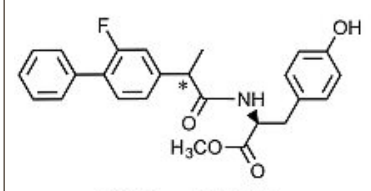

$(S, S)-\mathbf{2}$ and $(R, S)-\mathbf{2}$

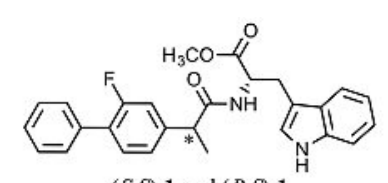

$(S, S)-\mathbf{1}$ and $(R, S)-1$

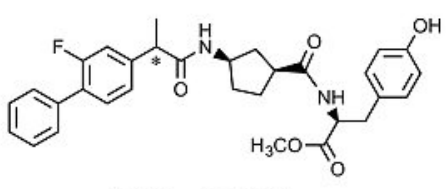

$(S, S)-3$ and $(R, S)-3$
Fig. 1. Chemical structures of the investigated systems.
Due to the complexity of the FBP/HSA system, the use of model dyads composed of FBP covalently linked to one specific amino acid has proven to be very useful to get information about the fundamental processes that may occur in the real supramolecular complexes. The picture obtained using such simplified model systems can be compared to the more complex drug/HSA systems. ${ }^{[7 \mathrm{~b}, 12 \mathrm{~h}, 12 \mathrm{k}, 18,22]}$

In this respect, we will herein discuss the photoreactivity of several model dyads composed of FBP and Trp or Tyr, where the two chromophores are directly linked through an amide bridge or separated by a cyclic spacer (see Fig. 1). The results will be compared to those obtained on the real FBP/HSA complex. We will in particular emphasize the contribution of time-resolved fluorescence measurements and how dynamical information adds to the global picture.

\section{Experimental}

\subsection{Steady-state}

Steady-state absorption spectra were recorded with a Perkin-Elmer Lambda 900 spectrophotometer. Steady-state fluorescence spectra were obtained using a JASCO spectrofluorometer system pro- vided with a monochromator in the wavelength range $200-900 \mathrm{~nm}$, with an excitation wavelength of $267 \mathrm{~nm}$ at $22^{\circ} \mathrm{C}$. Solutions were placed into $10 \times 10 \mathrm{~mm}$ quartz cells. The absorbance of the samples at the excitation wavelength was kept below 0.1 .

\subsection{Ultrafast Fluorescence Measure- ments}

Time-resolved fluorescence measurements were performed using the fluorescence upconversion (FU) and time-correlated single photon counting (TCSPC) techniques. ${ }^{[23]}$ The same excitation source was used for the two kinds of experiments: the third harmonic $(267 \mathrm{~nm})$ of a modelocked Ti-sapphire laser, delivering $\sim 120$ fs pulses whose repetition rate was 76 and 4.75 MHz for FU and TCSPC, respectively (in the latter case set by a pulse-picker).

For the FU measurements, a home-built setup was used. This has been described in detail earlier ${ }^{[24]}$ and a schematic view is given in Fig. 3. Briefly, a Ti:sapphire laser delivers the initial laser pulse (about 120 $\mathrm{fs}$ fwhm) at $800 \mathrm{~nm}$. This is then separated into two replicas by a beam-splitter. The first replica is converted into the UV 'pump' pulse by frequency-doubling and -tripling using two non-linear crystals (CNL1 and CNL2). This 'pump' pulse

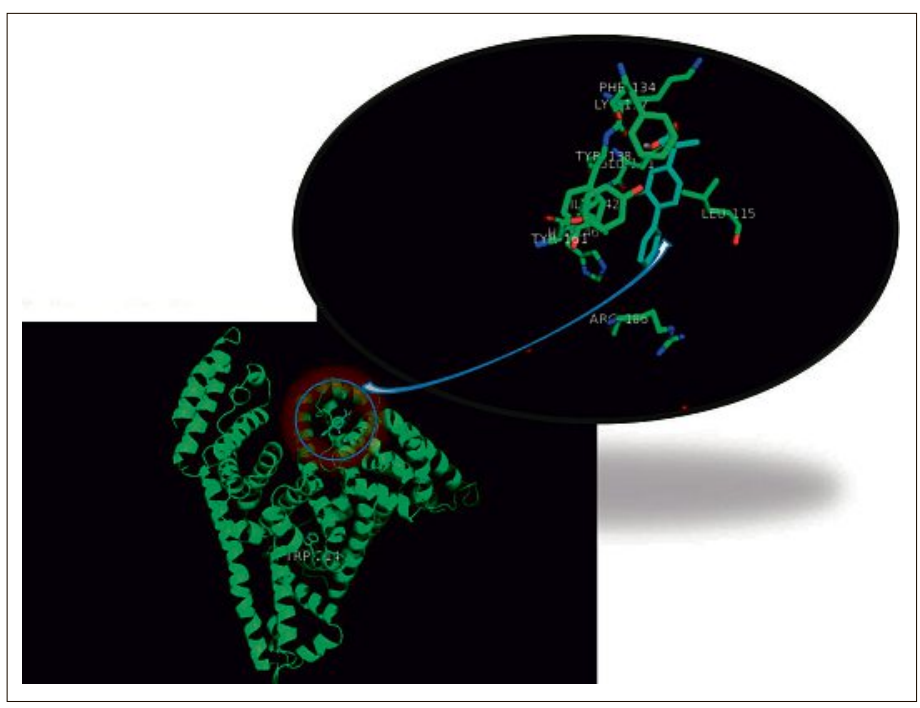

Fig. 2. Artistic view of a possible FBP binding to HSA. In this example, FBP binds to the IB subdomain of HSA. While the single tryptophan Trp-214 is fairly distant, two tyrosines, Tyr-138 and Tyr-161, are in close proximity. The picture has been prepared with the AutoDock software package $^{[21]}$ (http:// autodock.scripps. edu/resources/ references). 
excites the sample $(E)$ at a time $t=0$. The fluorescence emitted from the sample is collected and focused into a third nonlinear crystal (CNL3) where it is mixed with the second replica, the 'probe' pulse. The second replica, the 'probe' pulse, is delayed with respect to the 'pump' pulse by means of a delay line before being focused and superimposed with the fluorescence in the CNL3 non-linear crystal. In the present experiments a $1 \mathrm{~mm}$ type I BBO sum-frequency crystal (CNL3) was used for mixing the fluorescence and the fundamental laser pulse, providing an instrumental response function of about 350 fs (fwhm).

We judge that the time resolution of our FU setup is better than 100 fs after deconvolution, depending on the signalto-noise ratio. The average excitation power used was $40 \mathrm{~mW}$. The power density cannot be measured precisely within the excitation volume but we estimate it to 0.2 $\pm 0.1 \mathrm{GW} / \mathrm{cm}^{2}$ for a $40 \mathrm{~mW}$ output from the tripler unit (assuming a 40 micron diameter of the focused beam).

Solutions (about $30 \mathrm{~mL}$ ) were kept flowing through a $0.4 \mathrm{~mm}$ or $1 \mathrm{~mm}$ quartz cell, which was kept in continuous motion perpendicular to the excitation beam in order to minimize thermal effects. The concentration of the sample was adjusted to give an absorbance of about 0.5 at the excitation wavelength.

For the TCSPC experiments, a Becker \& Hickl GmbH SP-630 PC card was used.[26] The fluorescence from a standard quartz cell was collected and focused onto the entrance slit of a small monochromator (Jobin-Yvon HR250) using off-axis parabolic mirrors. In order to cut the laser light, a Schott WG 295 filter was placed in front of the slit. Moreover, a Glan-Thompson polarizer ensured that only the vertical component of the fluorescence was detected. The detector was a microchannel plate (R1564 U Hamamatsu) providing an instrumental response function of 60 ps (fwhm). The average laser power (0.1
$\mathrm{mW}$ at $4.75 \mathrm{MHz}$ ) was measured with a Melles Griot broadband powermeter. The irradiated area on the surface of the cell was $c a .0 .2 \mathrm{~cm}^{2}$ corresponding to a pulse intensity of $2.4 \mathrm{~kW} / \mathrm{cm}^{2}$. Solutions were contained in a $10 \mathrm{~mm} \times 10 \mathrm{~mm}$ quartz cell and continuously stirred. Successive recordings with the same sample gave identical decays, which were eventually merged to improve the signal-to-noise ratio. Such a procedure allowed us to ensure that the measured signals were not altered during the measurements due to a possible accumulation of photoproducts.

The time-resolved experiments (FU and TCSPC) were performed either at magic angle or under successive parallel $\left(I_{p a r}(t)\right)$ and perpendicular $\left(I_{\text {perp }}(t)\right)$ excitation/ detection conditions, defined as follows. Since only the vertical component of the emission was detected, in FU by the phase matching conditions of the crystal and in TCPSC by placing a Glan-Thomson polarizer in front of the monochromator, the parallel and perpendicular components are defined only by the polarization of the excitation beam. This was set to be either vertical or horizontal using a zero-order half-wave plate, mounted on a motorized rotation unit, allowing an easy adjustment.

From the measurements of the parallel and perpendicular components, the total (corresponding to magic angle) fluorescence and the fluorescence anisotropy were calculated from Eqns (1) and (2):

$$
\begin{aligned}
& F(t)=I_{\text {par }}(t)-G I_{\text {perp }}(t) \\
& r(t)=\frac{I_{\text {par }}(t)-G I_{\text {perp }}(t)}{I_{\text {par }}(t)+2 G I_{\text {perp }}(t)}
\end{aligned}
$$

The transmission of the excitation beam was found to be identical under parallel and perpendicular conditions so the correction factor $G$ was put to unity.

To evaluate the characteristic times involved, instead of treating $F(t)$ and $r(t)$ separately we performed a merged

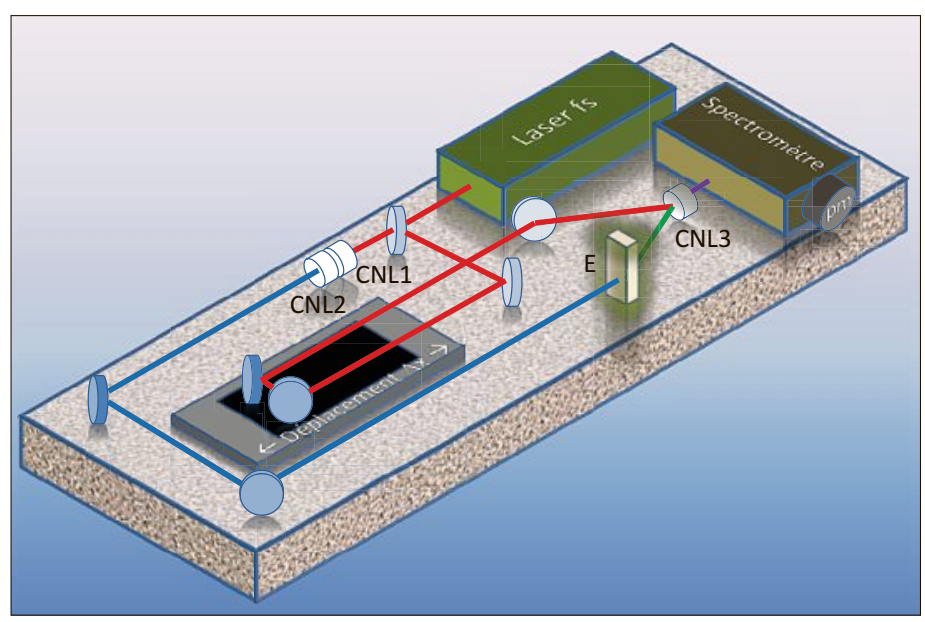

nonlinear fitting/deconvolution process using the impulse response model functions (Eqn. (3) and Eqn. (4))

$$
\begin{aligned}
& i_{\text {par }}(t)=(1+2 r(t)) f(t) \\
& i_{\text {perp }}(t)=(1-r(t)) f(t)
\end{aligned}
$$

convoluted by the Gaussian instrument response function, $I(t) \propto i(t) \otimes G(t)$. The model functions thus obtained were fitted to the experimentally measured $\left(I_{p a r}\right)$ and $\left(I_{\text {perp }}\right)$ signals. The full width at half maximum (fwhm) value of a Gaussian apparatus function was found to be about $375 \pm 12 \mathrm{fs}$ fwhm at $330 \mathrm{~nm}$.

\subsection{Docking Simulation}

The native structure of HSA was taken from the Protein Data Bank having PDB ID 1A06. Docking studies were performed with the AutoDock program suite. Autodock Vina and PyRx programs were employed to the virtual screening after which the docked models with varying binding energies were analysed using PyMol.

\section{Results}

\subsection{The Isolated Chromophores}

In order to use fluorescence measurements to draw any conclusions about molecular interactions in the dyads or the drug/protein complex, comparative data on the isolated non-interacting chromophores FBP (or amino acids) is needed.

Since we are here focusing on the ultrafast dynamics on the picosecond timescale, the FU decays of, on the one hand, FBP in acetonitrile, and, on the other hand, HSA and its intrinsic chromophores Trp and Tyr in PBS are shown in Figs. 4 and 5.

The fluorescence dynamics of FBP, shown in Fig. 4, display a rapid rise, limited by the temporal resolution, followed by a constant level, showing the absence of any rapid spectral evolution. Only in the blue wing, at $310 \mathrm{~nm}$, a slower rise of several picoseconds $(7.0 \pm 0.6 \mathrm{ps})$ is observed which is not present at the other wavelengths. This 'slow' rise can be assigned to a vibrational redistribution in the excited state. ${ }^{[12 \mathrm{k}]}$ The evolution of the fluorescence on a longer time-scale is characterized by a much slower decay on the nanosecond time-scale as evidenced by TCSPC measurements. This very 'stable' behaviour of the free FBP fluorescence makes it ideal for comparing with the behaviour in the dyad or the complex.

As can be seen in Fig. 5, the fluorescence decays of HSA and Trp at $340 \mathrm{~nm}$ follow each other closely during the first 10 picoseconds, characterized by a fast initial 
Fig. 4. Fluorescence decays of FBP in acetonitrile obtained by FU with $\lambda_{\text {exc }}=267$ $\mathrm{nm}$. The emission wavelengths are from top to bottom, 310 , $320,330,340,350$, 360 and $370 \mathrm{~nm}$.
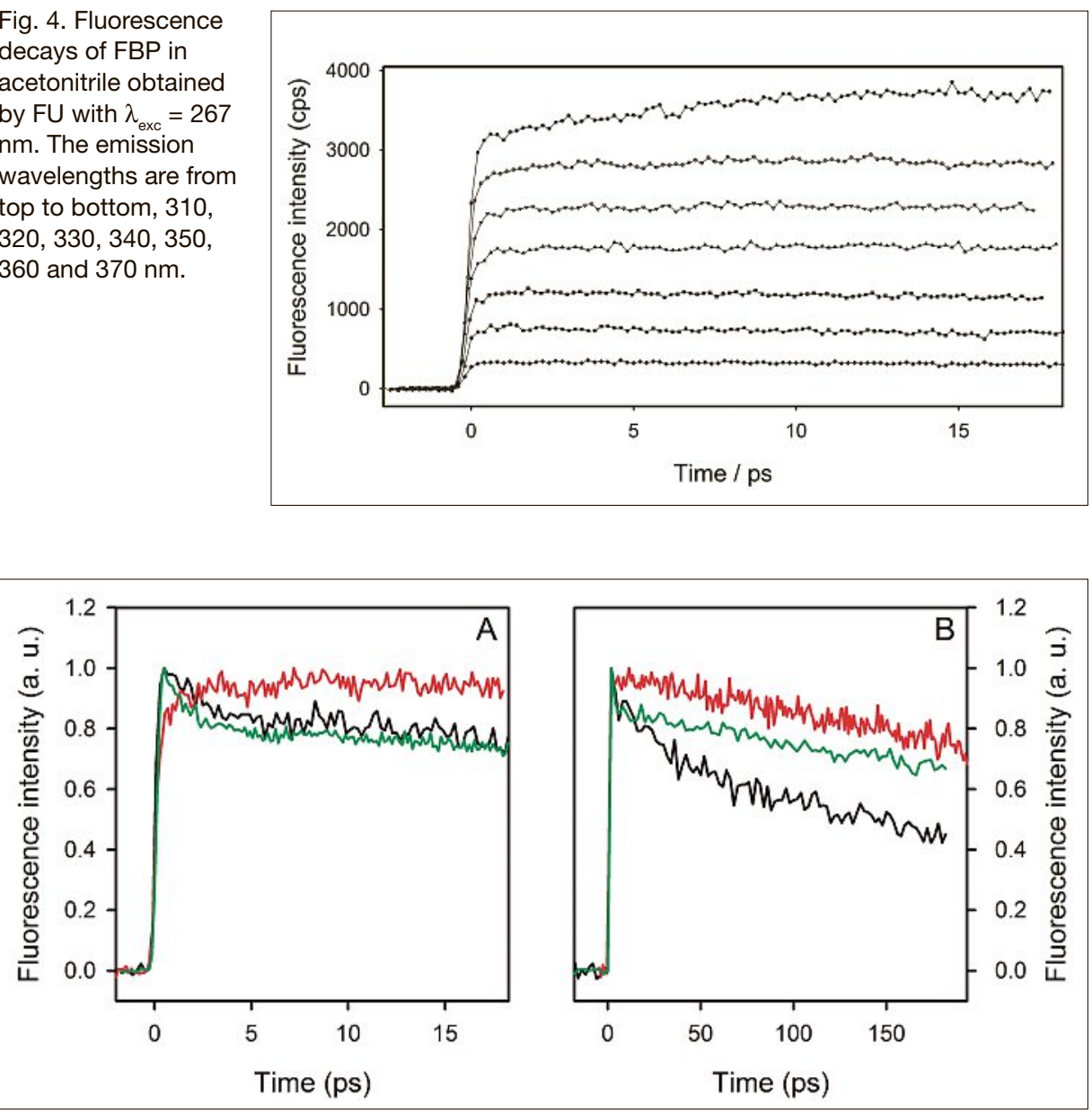

Fig. 5. Fluorescence decays of HSA/PBS at $340 \mathrm{~nm}$ (black), L-Tyr/PBS $310 \mathrm{~nm}$ (red) and L-Trp/ PBS at $340 \mathrm{~nm}$ (green) obtained by FU with $\lambda_{\text {exc }}=267 \mathrm{~nm}$.

decay before levelling off to a slower decay rate. This is not surprising since the protein emission is known to be completely dominated by the tryptophan emission. Beyond 10 picoseconds, however, the HSA fluorescence intensity decays faster than that of Trp but in a non-linear manner. Beyond 150 picoseconds the two curves seem to evolve with the same slope. The tyrosine fluorescence decay at $310 \mathrm{~nm}$, on the other hand, is characterised by a rapid rise before starting to decay with an apparently similar rate as the protein and Trp.

\subsection{Model FBP-Trp Dyads}

Diastereomeric dyads composed of $(S)$ - or $(R)$-FBP covalently linked to $(S)$ Trp through an amide bridge $((S, S)-\mathbf{1}$ and $(R, S)$-1), see Fig. 1) were synthesised by conventional methods. ${ }^{[22 \mathrm{~d}]}$ Experiments were performed in acetonitrile due to the low solubility of the linked systems in aqueous media. The UV absorption spectra of dyads $\mathbf{1}$ matches the added spectra of the isolated chromophores at the same concentration (see Fig. 6A), indicating the absence of significant interactions between the two partners in the groundstate. Fluorescence measurements of isoabsorptive solutions of FBP and dyads wavelength band around $450 \mathrm{~nm}$, assigned to exciplex emission, was also detected; this band was higher for $(R, S)-\mathbf{1}$ (see inset Fig. 6B).

The efficient FBP quenching can be explained by an energy transfer process from ${ }^{1} \mathrm{FBP} *$ to $\operatorname{Trp},{ }^{[12 \mathrm{k}, 22 \mathrm{~d}]}$ which is in line with the higher excited state energy of FBP (99 $\mathrm{kcal} \mathrm{mol}^{-1}$ ) compared to Trp (96 kcal $\left.\mathrm{mol}^{-1}\right){ }^{[12 \mathrm{a}, 27]}$ Fluorescence decays were recorded by FU and TCSPC at 310 and $340 \mathrm{~nm}$ (see Fig. 7). In general, the decay kinetics of the dyads were much faster than those of the isolated drug or amino acid; the kinetic results are a clear indicative of the dynamic nature of the fluorescence quenching.

The FU decays at $310 \mathrm{~nm}$, where emission is dominated by FBP, displayed an instantaneous rise followed by a rapid decay on the picosecond time scale. This behaviour can be attributed to a strong drug-amino acid coupling. Stereodifferentiation was noticed as the kinetic trace of $(R, S)$-1 decayed faster than that of $(S, S)-\mathbf{1}$. By contrast, a rise of several picoseconds ( $\mathrm{ca}$. $7.0 \pm 0.6 \mathrm{ps}$ ), assigned to a vibrational redistribution in the excited state, and slower decay was detected for FBP (see Fig. 7A). ${ }^{[12 k]}$ The FU decays at $340 \mathrm{~nm}$, where Trp emission dominates, were slower than those at $310 \mathrm{~nm}$. The kinetic traces of the dyads decayed faster than that of Trp, and again exhibited a clear stereodifferentiation, showing higher quenching for the $(R, S)$-diastereomer (see Fig. 7B). The kinetic results are in line with the steady-state fluorescence data.

In order to further characterise the dynamics of the investigated systems, TCSPC measurements were performed. The decays profiles at 310 and $340 \mathrm{~nm}$ on the nanosecond time range followed the same trend than the FU data, that is faster decay for the dyads compared to the isolated chromophores, the fastest being for $(R, S)$-1. However, the kinetic traces at

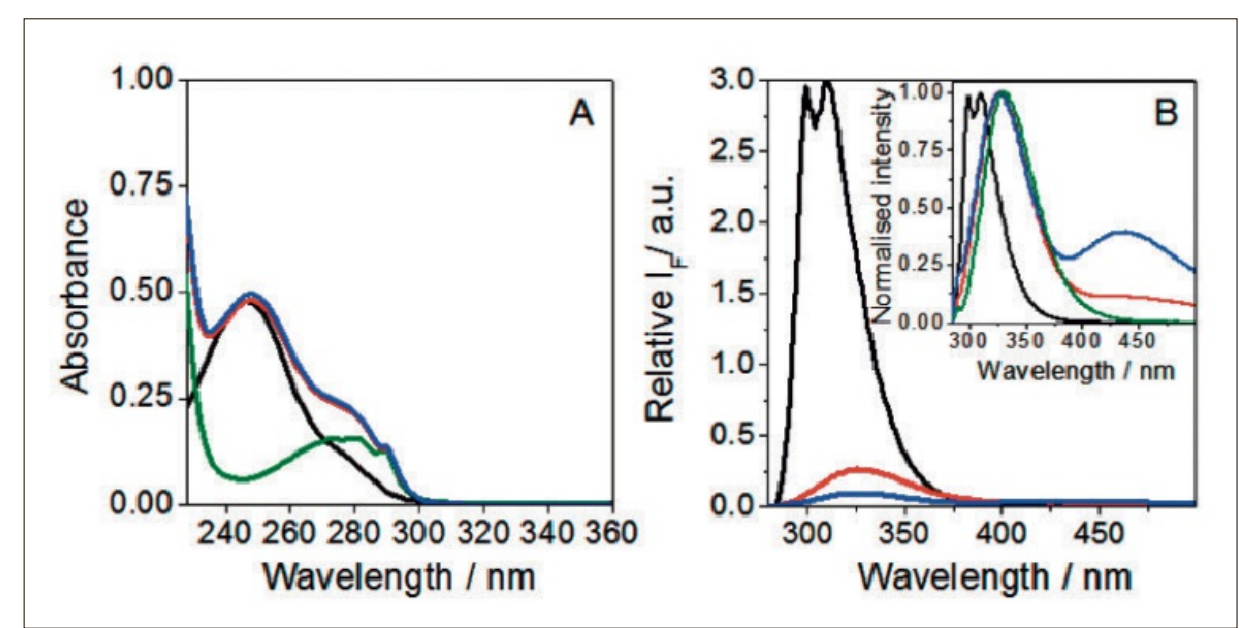

Fig. 6. UV absorption spectra $(25 \mu \mathrm{M}) \mathrm{A})$ and steady-state emission $\left(\lambda_{\text {exc }}=267 \mathrm{~nm}\right)$ of isoabsorptive solutions B) of FBP (black), Trp (green), $(S, S)-1$ (red) and $(R, S)-1$ (blue) in deaerated acetonitrile. The inset shows the normalised emission. Reproduced by permission of The Royal Society of Chemistry (Phys. Chem. Chem. Phys. 2013, 15, 4727; DOI: 10.1039/c3cp43847c). ${ }^{[12 k]}$ 


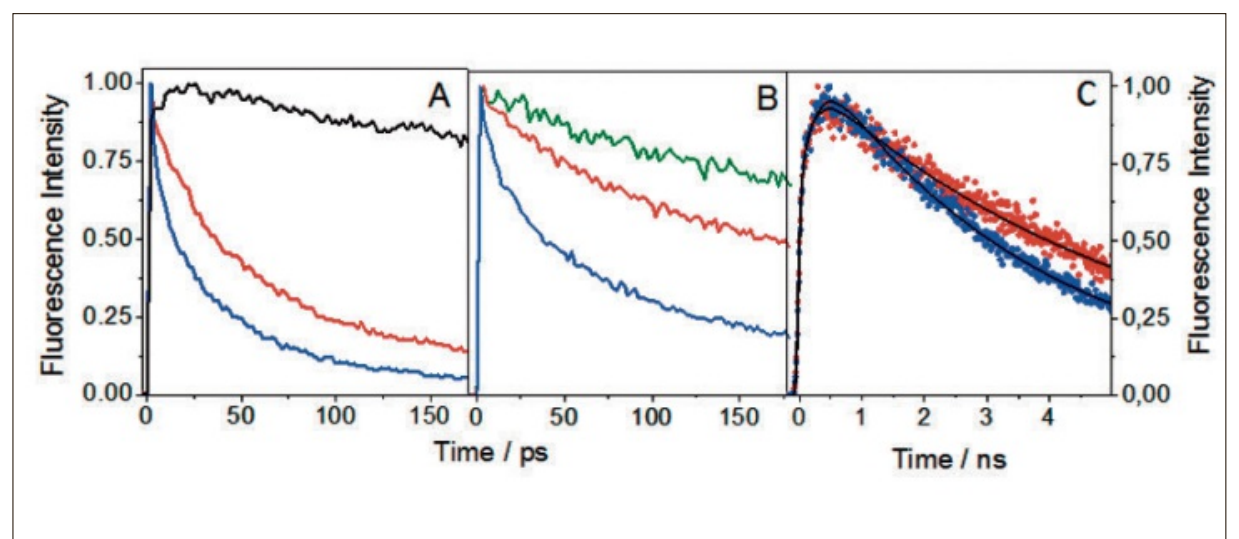

Fig. 7. Normalised FU decays at A) $\lambda_{\text {em }}=310 \mathrm{~nm}$ and B) $\lambda_{\text {em }}=340 \mathrm{~nm}$ of FBP (black), Trp (green), $(S, S)-1$ (red) and (R,S)-1 (blue). C) Normalised TCSPC decays at $\lambda_{\text {em }}=450 \mathrm{~nm}$ of $(S, S)-1$ (red) and $(R, S)-1$ (blue); fitted curves are shown in black. All measurements were performed in acetonitrile. Reproduced by permission of The Royal Society of Chemistry (Phys. Chem. Chem. Phys. 2013, 15, 4727; DOI: $10.1039 / \mathrm{c} 3 \mathrm{cp} 43847 \mathrm{c}) .{ }^{[12 k]}$

$450 \mathrm{~nm}$, where exciplex emission was detected in the steady-state experiments, were much slower (see Fig. 7C). Interestingly, these traces are characterised by arise with time values $c a .115 \pm 7 \mathrm{ps}$ and $189 \pm 6$ ps for $(S, S)-\mathbf{1}$ and $(R, S)-\mathbf{1}$, respectively, ${ }^{[12 \mathrm{k}]}$ which is attributed to the formation of the exciplex states. Their lifetimes were 5.34 $\pm 0.02 \mathrm{~ns}$ and $3.63 \pm 0.01 \mathrm{~ns}$ for the $(S, S)$ and $(R, S)$-diastereomers, respectively. Such exciplexes are intermediate states for full electron transfer process, ${ }^{[28]}$ consistent with the electron donor character of Trp. ${ }^{[10]}$

Fluorescence anisotropy decays at 310 $\mathrm{nm}$ were longer for the dyads than for the parent drug, and decayed following a mono-exponential law. The anisotropy lifetime for FBP was found to be $c a$. 26 $\pm 1 \mathrm{ps}$, while for $(S, S)-\mathbf{1}$ and $(R, S)-\mathbf{1}$ were ca. $42 \pm 2$ ps and $44 \pm 4$ ps, respectively. This behaviour can be easily understood in terms of the larger hydrodynamic volume of the dyads. The anisotropy profiles at 340 $\mathrm{nm}$ were also determined; the characteristic times were $24 \pm 1$ ps and $32 \pm 2$ ps for $(S, S)-\mathbf{1}$ and $(R, S)-\mathbf{1}$, respectively, which is significantly faster than what was observed at $310 \mathrm{~nm}$; this can be the result of the direct excitation of $\operatorname{Trp}\left(\mathrm{r}_{0}=0.17 \pm 0.01\right)$ without the contribution from $\mathrm{FBP}\left(\mathrm{r}_{0}=0.31 \pm\right.$ $0.01)$. Indeed, the zero time fluorescence anisotropies for $(S, S)-\mathbf{1}$ and $(R, S)-\mathbf{1}$ at $340 \mathrm{~nm}$ were $0.25 \pm 0.01$ and $0.22 \pm 0.01$, respectively, representing average values of FBP and Trp. ${ }^{[12 k]}$ Finally, the anisotropy decay lifetimes at $450 \mathrm{~nm}$ were found to be zero at all times for both diastereomers, contrary to those recorded at 310 and 340 $\mathrm{nm}$. This points to the different nature of the excited state emitting at $450 \mathrm{~nm}$, strengthening the assignment of the long wavelength emission to exciplex states.

Regarding the Trp fluorescence quenching mechanism, observed in the steady state and time-resolved measurements, it could involve either electron transfer (ET) jphotochem.2016.03.006). ${ }^{[30]}$

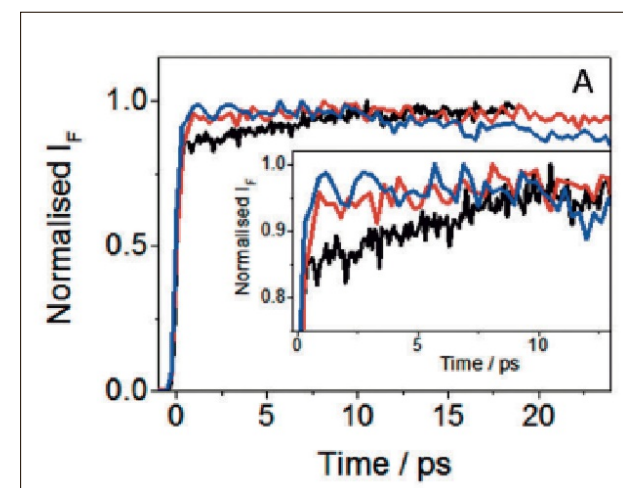
jphotochem.2016.03.006). ${ }^{[30]}$ or exciplex formation (EXC). Application of the Weller equations allowed us to estimate the $\Delta \mathrm{G}$ values corresponding to the two possible pathways. ${ }^{[29]}$ Both processes were found to be exergonic, with $\Delta \mathrm{G}_{\mathrm{ET}}$ $=-15 \mathrm{kcal} \mathrm{mol}^{-1}$ and $\Delta \mathrm{G}_{\mathrm{EXC}}=-10 \mathrm{kcal}$

$\mathrm{mol}^{-1}$, respectively. Actually, exciplexlike states were directly observed for both dyads.

\subsection{Model FBP-Tyr Dyads}

Diastereomeric dyads $\mathbf{2}$ and $\mathbf{3}$ were again synthesised following established methods. ${ }^{[30]}$ In dyads 2, FBP and Tyr were directly linked while in dyads $\mathbf{3}$ they were separated by a cyclic spacer. Following the same methodology as for diastereomers 1, all measurements were performed in acetonitrile. Like in the case of the FBPTrp dyads, the UV absorption spectra of the FBP-Tyr dyads were identical to added spectra of the isolated chromophores at the same concentration $(25 \mu \mathrm{M})$, indicative of the absence of significant interactions between the two units in the ground state.

Fluorescence spectra of isoabsortive solutions of FBP, $\mathbf{2}$ and $\mathbf{3}$ at $\lambda_{\text {exc }}=267 \mathrm{~nm}$, where $90 \%$ of the photons are absorbed by the drug, are shown in Fig. 8. For the dyads, the spectra are dominated by the FBP emission. This is in line with its lower singlet excited state energy $(99 \mathrm{kcal}$ $\left.\mathrm{mol}^{-1}\right)$ compared to that of Tyr $(100 \mathrm{kcal}$

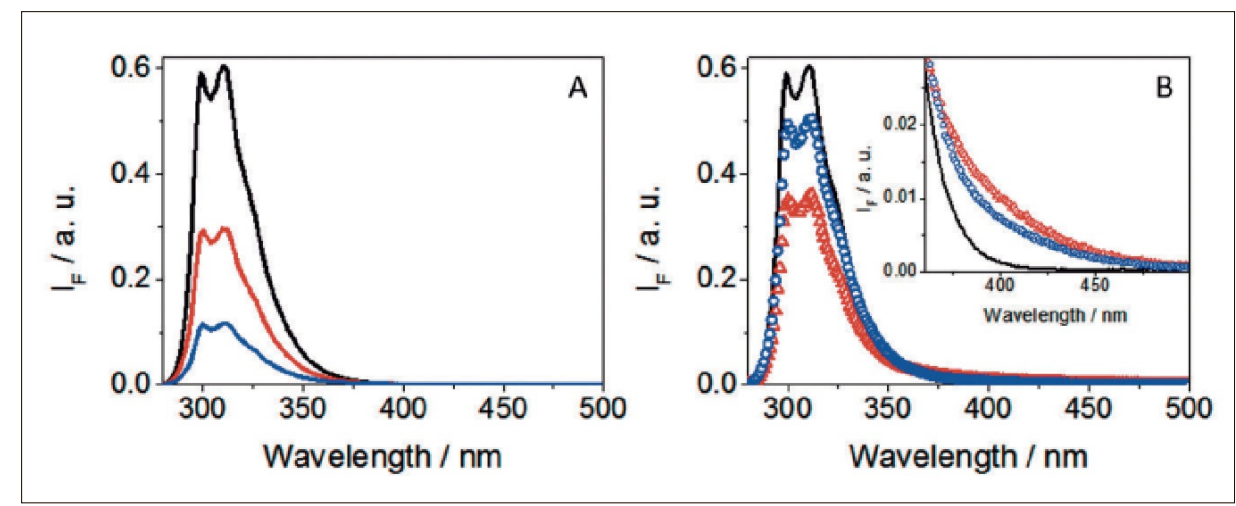

Fig. 8. Fluorescence spectra of A) (S)-FBP (black line), (S,S)-2 (red line) and (R,S)-2 (blue line), and B) (S)-FBP (black line), $(S, S)-3$ (red triangles) and $(R, S)-3$ (blue circles) after excitation at $267 \mathrm{~nm}$ in deaerated MeCN. The inset shows emission from exciplex states for dyads 3 . Reproduced by permission of Elsevier (J. Photochem. Photobiol. A 2016, 322, 95; http://dx.doi.org/10.1016/j.

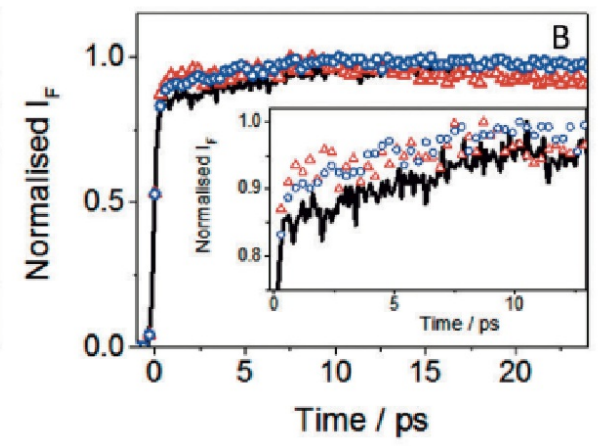

Fig. 9. Normalised FU decays at $\lambda_{\text {mon }}=310 \mathrm{~nm}$ for A) (S)-FBP (black line), ( $\left.S, S\right)-2$ (red line) and $(R, S)$-2 (blue line), and B) (S)-FBP (black line), (S,S)-3 (red triangles) and ( $R, S)$-3 (blue circles) after excitation at $267 \mathrm{~nm}$ in MeCN. The insets show a zoom of the decay traces. Reproduced by permission of Elsevier (J. Photochem. Photobiol. A 2016, 322, 95; http://dx.doi.org/10.1016/j. 
$\left.\mathrm{mol}^{-1}\right)$. $^{[12 \mathrm{a}, 29 \mathrm{a}]}$ Interestingly, quenching of ${ }^{1} \mathrm{FBP}^{*}$ was noticed for all dyads, being more efficient for $\mathbf{2}$ than for $\mathbf{3}$ (see Fig. $8)$. The process was stereoselective, being higher for the $(R, S)$-diastereomer in the case of the directly linked chromophores while the opposite trend was observed for the separated ones. Strikingly, a tiny but significant emission tail at long wavelengths $(>350 \mathrm{~nm})$ which can be assigned to exciplex emission, was detected for the spacer separated dyads $\mathbf{3}$, but not for the directly linked dyads $\mathbf{2}^{[31]}$ In line with the ${ }^{1} \mathrm{FBP}^{*}$ quenching, this emission is higher for the $(S, S)$-derivative.

FU decays $\left(\lambda_{\text {exc }}=267\right) \mathrm{nm}$ are shown in Fig. 9. A rise in the first picoseconds was detected for dyads $\mathbf{3}$, similar to that previously observed for FBP (see Fig. 9B) and assigned to vibrational relaxation. However, no such rise was observed for dyads 2 (see Fig. 9A). The reason for this relatively minor difference is not clear, but it only concerns a smaller part of the excited dyads. On the one hand, it may be attributed to a stronger drug-amino acid coupling in the directly linked systems $\mathbf{2}$, leading to a fast quenching that compensates the rise of the ${ }^{1}$ FBP* emission. On the other, it may be rapidly quenched tyrosine fluorescence, compensating the rise of the ${ }^{1} \mathrm{FBP}^{*}$ emission. Geometry considerations (see below) lead us to favour the first possibility. No stereoselectivity was detected at early times, but appears after about 10 ps.

The kinetic of $(R, S)-2$ starts to decay faster than its $(S, S)$-analogue after $10 \mathrm{ps}$; by contrast, for the dyads 3 the opposite behaviour is observed. This trend persists at longer time scales. Indeed, TCSPC measurements showed that the ${ }^{1} \mathrm{FBP} *$ lifetime decreased from $1.67 \mathrm{~ns}$ for the isolated drug to 0.90 and 0.36 ns for $(S, S)-2$ and $(R, S)-\mathbf{2}$, respectively. By contrast, dyads $\mathbf{3}$ showed lifetimes $\mathrm{ca}$. 0.93 and $1.20 \mathrm{~ns}$ for $(S, S)-\mathbf{3}$ and $(R, S)-\mathbf{3}$, respectively; the fluorescence lifetimes of the exciplex emission at $420 \mathrm{~nm}$ were also measured. [12k,32] It turns out to be much more longer-lived than at $310 \mathrm{~nm}$. The fluorescence profiles are characterised by a rise of $0.38 \pm 0.01$ ns and $0.69 \pm 0.01 \mathrm{~ns}$ and a decay of 3.27 $\pm 0.01 \mathrm{~ns}$ and $3.05 \pm 0.01 \mathrm{~ns}$ for $(S, S)-3$ and $(R, S)-3$, respectively.

Fluorescence anisotropies measured at $310 \mathrm{~nm}$ displayed longer decays for dyads 2 and $\mathbf{3}$ ( $\mathrm{ca} .50 \pm 4 \mathrm{ps}$ ) compared to FBP $(26 \pm 1 \mathrm{ps})$. This is in full agreement with the larger hydrodynamic volumes of the dyads compared to the isolated drug. The high $\mathrm{r}_{0}$ values for all dyads ( $c a$. 0.35 \pm 0.02 ) indicate that the emission arose from the directly excited state of the drug $\left(r_{0}=0.34 \pm 0.02\right)$, with no change in their electronic nature.

Following the same procedure as for dyads $1, \Delta \mathrm{G}$ values for electron transfer or exciplex formation were calculated to determine the mechanism of ${ }^{1} \mathrm{FBP} *$ quenching for dyads 2 and 3 . Both processes were found to be exergonic with $\Delta \mathrm{G}_{\mathrm{ET}}=-9 \mathrm{kcal} \mathrm{mol}^{-1}$ and $\Delta \mathrm{G}_{\mathrm{EXC}}=-4$ $\mathrm{kcal} \mathrm{mol}^{-1}$. However, as mentioned above, exciplex emission was detected for dyads 3, implying that electron transfer is at least partly responsible for the ${ }^{1} \mathrm{FBP} *$ quenching in this case.

Finally, PM3 molecular modelling was performed with the aim of clarifying the differences in the stereoselective quenching observed in dyads $\mathbf{2}$ and $\mathbf{3}$. The higher fluorescence quenching for $\mathbf{2}$ compared to 3 was evident due to the better coupling between FBP and Tyr in the directly linked systems (see Fig. 10). Besides, a better alignment was observed for $(R, S)$ 2 compared to the $(S, S)$-analogue, which is in line with its stronger quenching. By contrast, the geometrical arrangement for $(R, S)$-3 was much more distorted than that of $(S, S)-3$, in agreement with its lower quenching.

\subsection{FBP/HSA Complexes}

Spectroscopic measurements were performed on mixtures of $(S)$ - or $(R)$ FBP $(25 \mu \mathrm{M})$ and HSA $(36 \mu \mathrm{M})$ in PBS; [12k] under these conditions, all the drug is bound to the protein. ${ }^{[12 \mathrm{~d}, 19]}$ The fluorescence spectra obtained with $\lambda_{\text {exc }}=$ $267 \mathrm{~nm}$ of the isolated (S)-FBP and HSA as well as the $(S)$-FBP/HSA mixtures are shown in Fig. 11A (similar results were obtained for the $(R)$-enantiomer; data not shown). The results reveal that both drug and protein contribute to the emission after the complex formation. A significant fluorescence quenching was detected for FBP/HSA, but to a lower extent and with a lower stereoselectivity than for the dyads, probably due to the weaker interaction in the non-covalent supramolecular complexes.
Considering the relative absorbances of drug and protein at the excitation wavelength (18\% of the photons are absorbed by FBP and $82 \%$ by HSA at $267 \mathrm{~nm}$ ), and assuming independent emission, the fluorescence spectrum of $\mathrm{FBP} / \mathrm{HSA}$ mixtures can be determined as:

$$
\begin{aligned}
\mathrm{A}_{\mathrm{F}}(\mathrm{tot})= & 0.18 \times \mathrm{A}_{\mathrm{F}}(\mathrm{FBP})+0.82 \\
& \times \mathrm{A}_{\mathrm{F}}(\mathrm{HSA})
\end{aligned}
$$

where $A_{F}(F B P)$ and $A_{F}(H S A)$ are the individual fluorescence spectra of isoabsorptive solutions of FBP and HSA at the excitation wavelength $(267 \mathrm{~nm})$. However, this simulated spectrum (dark red line in Fig. 11A) did not match the experimental one (red line in Fig. 11A). Instead, a 'best' reproduction of the real drug/protein fluorescence spectrum (violet line in Fig. 11A) was achieved using the relation:

$$
\begin{aligned}
\mathrm{A}_{\mathrm{F}}(\text { tot })= & 0.074 \times \mathrm{A}_{\mathrm{F}}(\mathrm{FBP})+0.746 \\
& \times \mathrm{A}_{\mathrm{F}}(\mathrm{HSA})
\end{aligned}
$$

indicating a noticeable quenching of the FBP emission as well as a slight quenching of the protein emission.

Fluorescence decays were recorded for FBP, HSA, $(S)$-FBP/HSA and $(R)-\mathrm{FBP} /$ HSA in PBS with $\lambda=267 \mathrm{~nm}$ using both FU and TCSPC. In analogy with what was observed in acetonitrile, the fluorescence of FBP in PBS measured by $\mathrm{FU}$ at $310 \mathrm{~nm}$ shows a slight rise during the first few picoseconds. This is in line with an intramolecular vibrational relaxation process. For the FBP/HSA mixtures, on the other hand, the traces measured at $310 \mathrm{~nm}$, where the FBP emission is dominating, displays an instantaneous rise. This can be attributed to encapsulation of the drug within the protein cavities, resulting in a reduced coupling of its vibrational modes. During the first few

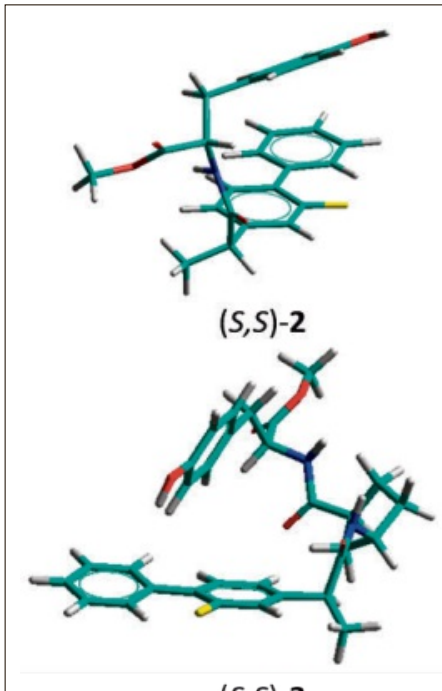

$(S, S)-3$

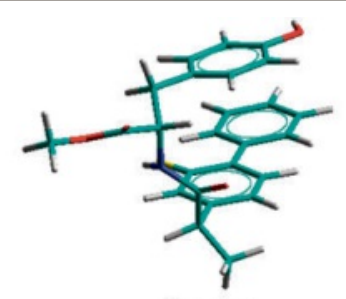

$(R, S)-2$

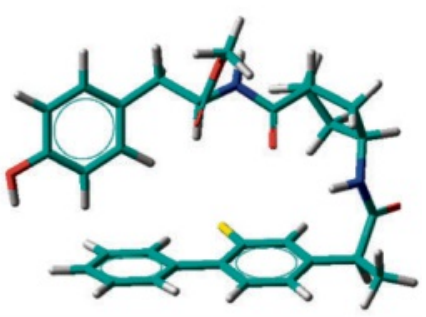

$(R, S)-3$
Fig. 10. Geometry optimised (HyperChem Release 8.0.3 for Windows Molecular Model System, PM3) structures for all investigated dyads. Reproduced by permission of Elsevier (J. Photochem. Photobiol. A 2016, 322, 95; http://dx.doi. org/10.1016/j.jphotochem.2016.03.006). ${ }^{[30]}$ 


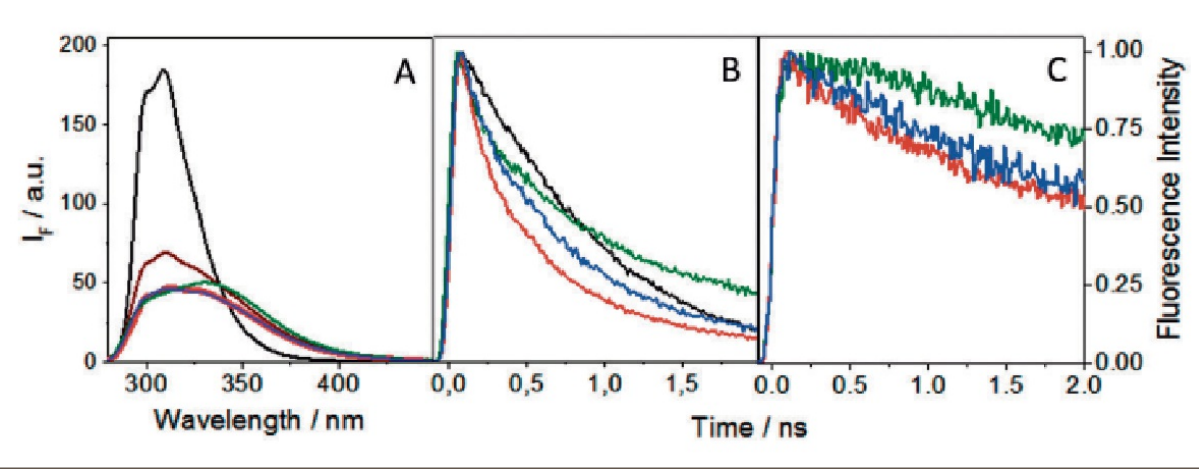

Fig. 11. A) Fluorescence spectra of (S)-FBP (black), HSA (green), and (S)-FBP/HSA (red) in PBS, under air, using isoabsorptive solutions at the excitation wavelength $(267 \mathrm{~nm})$. The simulated fluorescence spectrum, considering only the percentage of light absorbed by each subunit, is shown in dark red. The 'best' simulated fluorescence spectrum, as explained in the text, is shown in violet. B) Normalised TCSPC decays of (S)-FBP (black), HSA (green), (S)-FBP/HSA (red) and $(R)$-FBP/HSA (blue) in PBS at the maximum emission $\left(\lambda_{\text {em }}=310 \mathrm{~nm}\right.$ for FBP and FBP/HSA mixtures, and $\lambda_{\mathrm{em}}=340 \mathrm{~nm}$ for HSA). C) Normalised TCSPC decays as in B) but at $\lambda_{\mathrm{em}}=380 \mathrm{~nm}$. Reproduced by permission of The Royal Society of Chemistry (Phys. Chem. Chem. Phys. 2013, 15, 4727; DOI: 10.1039/c3cp43847c). .12k] $^{[2]}$

picoseconds the fluorescence decays of the FBP/HSA complexes are similar to that of FBP, and after several tens of picoseconds they started to decay faster than that of the free drug. A certain stereoselectivity was observed, with the $(S)$-FBP/HSA fluorescence decaying faster than its $(R)$ analogue.

This stereoselective dynamic quenching persists at longer times, as evidenced by the TCSPC fluorescence profiles shown in Figs. 11B and 11C. While the kinetic profile of FBP decayed following a monoexponential function, those of HSA and FBP/HSA were highly nonexponential and wavelength dependent; a three-exponential model function $(\mathrm{f}(\mathrm{t})$ $=\mathrm{a}_{1} \cdot \exp \left(-\mathrm{t} / \tau_{1}\right)+\mathrm{a}_{2} \cdot \exp \left(-\mathrm{t} / \tau_{2}\right)+\mathrm{a}_{3} \cdot \exp (-\mathrm{t} /$ $\left.\tau_{3}\right)$ ) was necessary to get a good fit of the kinetic traces. This is not surprising due to the complex protein structure. Thus, the average lifetime $(<\tau\rangle=\mathrm{a}_{1} \tau_{1}+\mathrm{a}_{2} \tau_{2}+$ $\mathrm{a}_{3} \tau_{3}$ ), was used to make the comparisons easier. At $310 \mathrm{~nm}$ (Fig. 11B), the FBP fluorescence decayed with a lifetime of $0.78 \mathrm{~ns}$, while the HSA fluorescence decayed with an average lifetime of 1.22 ns. The fluorescence kinetics of the FBP/ HSA mixtures at $310 \mathrm{~nm}$ were slightly faster than those of FBP or HSA at their maximum emission. This quenching was more pronounced for the $(S)$-enantiomer $(<\tau>=0.54 \mathrm{~ns})$ than for $(R)$-FBP/HSA $(<\tau>$ $=0.70 \mathrm{~ns}$ ). At $380 \mathrm{~nm}$ (Fig. 11C), where only HSA emits, the fluorescence decays become substantially longer. Still, the $\langle\tau>$ values were shorter for the complexes (3.69 ns for $(S)$-FBP/HSA and $4.14 \mathrm{~ns}(R)$ FBP/HSA) compared to that of HSA (5.78 ns). Again, a clear stereoselectivity was detected. Interestingly, both FBP and Trp emissions were quenched in the FBP/HSA complex, but less than in the covalently linked dyads.
The time-dependent fluorescence anisotropies were also recorded for FBP, HSA and FBP/HSA under the same conditions as above using FU and TCSPC. The resulting fluorescence anisotropy decays are shown in Fig. 12.

The initial fluorescence anisotropy of HSA $\left(r_{0}=0.18 \pm 0.02\right)$, is much lower than the theoretical limit of 0.4 for parallel absorption and emission transition dipoles. This can be taken as an indication for complex internal relaxation processes and/or energy transfer preceding the tryptophan emission. The fluorescence anisotropy remained practically constant during the first hundreds of picoseconds, in line with a slow rotational diffusion of the voluminous protein.

The initial fluorescence anisotropy of free FBP is high, $r_{0}=0.36 \pm 0.02$, and decays rapidly, with a characteristic time of $65 \pm 6 \mathrm{ps}$, in accordance with free diffusional rotation.

In contrast, when FBP is complexed with the protein, the initial fluorescence anisotropy dropped to $0.29 \pm 0.02$ and the kinetics remained constant in the first 150 ps. This can be explained as due to a more constrained environment provided by the protein binding sites, limiting the degrees of freedom for conformational relaxation of the drug.

Interestingly, at longer times the fluorescence anisotropy decays of the FBP/HSA complexes revealed a clear stereoselectivity (Fig. 12B). They decay monoexponentially, with characteristic lifetimes of $0.44 \pm 0.03 \mathrm{~ns}$ and $0.62 \pm$ $0.07 \mathrm{~ns}$ for $(S)$-FBP/HSA and $(R)$-FBP/ HSA, respectively, but to a constant level $\mathrm{r}_{\infty}>0$, indicating a restricted rotational motion ( $c f$. wobble-in-a-cone model). The different lifetimes and level $r_{\infty}$ values can be related to the different dynamics of the two enantiomers within the binding pocket. One can deduce that the reorientational relaxation is more efficiently restricted for the $(R)$-enantiomer than for $(S)$-FBP.

\section{Conclusions}

The photoreactivity of the nonsteroidal anti-inflammatory drug flurbiprofen to human serum albumin has been studied in terms of different covalent model dyads composed of FBP and Trp or Tyr, two amino acids that play a key role in the interaction of a drug to HSA, and in the real supramolecular system. In general, dynamic fluorescence quenching has been detected, with a clear stereoselectivity that varied depending on the conformation

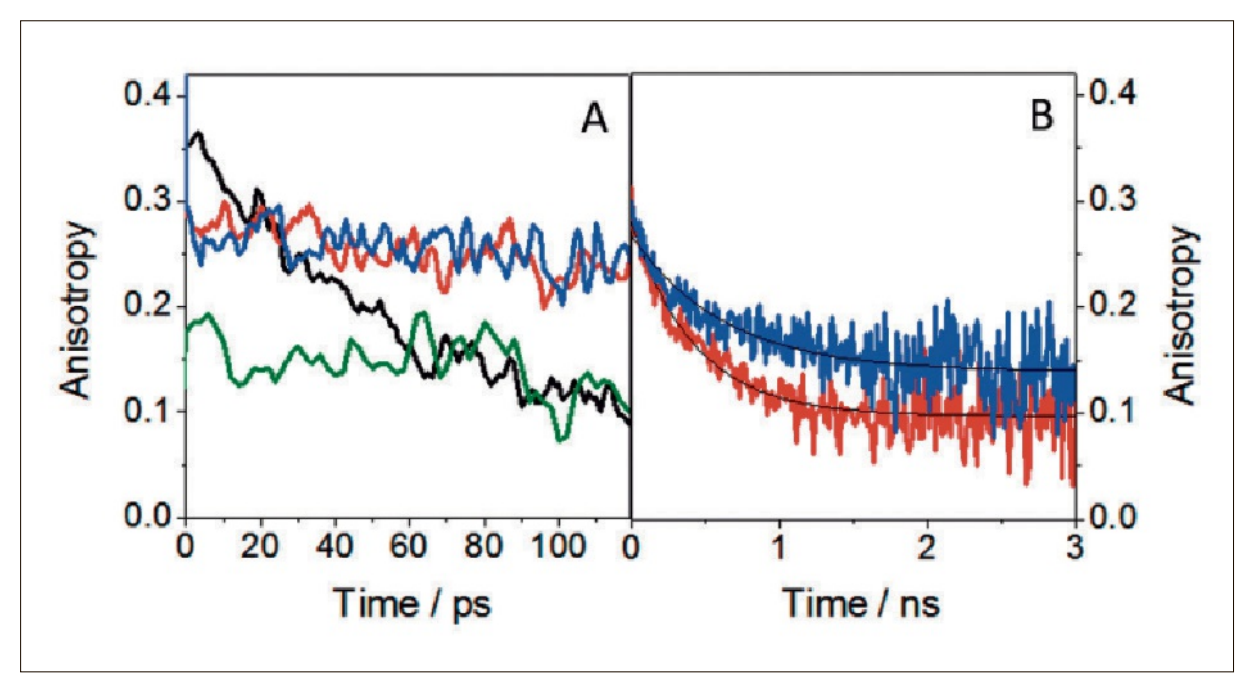

Fig. 12. Fluorescence anisotropy decays of (S)-FBP (black), HSA (green), (S)-FBP/HSA (red) and (R)-FBP/HSA (blue) in PBS at $\lambda_{\text {em }}=310 \mathrm{~nm}$. A) FU and B) TCSPC after excitation at $267 \mathrm{~nm}$ (the best fit is shown in black solid line). Reproduced by permission of The Royal Society of Chemistry (Phys. Chem. Chem. Phys. 2013, 15, 4727; DOI: 10.1039/c3cp43847c). ${ }^{[12 k]}$ 
arrangement of the investigated system. The $(R, S)$-diastereomer evidenced higher quenching than its $(S, S)$-analogue in FBP-Trp dyads. A similar tendency was detected for the directly linked FBP-Tyr models, while the opposite behaviour was observed when FBP and Tyr were separated by a cyclic spacer. Application of the Weller equation was in agreement with electron transfer and exciplex formation as the thermodynamically favourable routes for the fluorescence quenching. Molecular modelling results were in agreement with the stereoselectivity observed for FBPTyr dyads. Finally, the same fundamental processes occurred in the dyads and in the real FBP/HSA complexes, although on different rates and time scales; thus, the dynamic quenching rates were slower in the supramolecular system most probably due to the weaker non-covalent drug/ protein interactions compared to the dyads. In order to get a clearer picture of the FBP/HSA interaction, molecular docking simulations could bring valuable information about the actual binding sites, where FBP may interact locally with several other residues. Studying covalently bound dyads formed by FBP and the amino acids involved at the binding sites by timeresolved fluorescence spectroscopy would surely bring a more detailed understanding of the structure and the dynamics of the FBP/HSA complex.

\section{Acknowledgements}

Financial support from the Spanish Government (CTQ2013-47872-C2-1-P), EU (MSCA-657465 and LASERLAB-EUROPE grant agreement no. 284464) and Generalitat Valenciana (PROMETEOII/2013/005) is gratefully acknowledged.

Received: January 13, 2017

[1] a) T. Peters, in 'All About Albumin Biochemistry, Genetics, and Medical Applications', Ed. Elsevier, Academic Press, San Diego, 1995, p. 76; b) S. Sugio, A. Kashima, S. Mochizuki, M. Noda, K. Kobayashi, Protein Eng. 1999, 12, 439.

[2] a) U. Kragh-Hansen, V. T. G. Chuang, M. Otagiri, Biol. Pharm. Bull. 2002, 25, 695; b) T Peters, in 'Advances in Protein Chemistry', Vol. 37, Academic Press, New York, 1985, p. 161; c) K. Vuignier, J. Schappler, J.-L. Veuthey, P.-A. Carrupt, S. Martel, Anal. Bioanal. Chem. 2010 $398,53$.

[3] S. Schmidt, D. Gonzalez, H. Derendorf, $J$. Pharm. Sci. 2010, 99, 1107.

[4] D. C. Carter, J. X. Ho, in 'Advances in Protein Chemistry', Vol. 45, Academic Press, New York, 1994, p. 153.

[5] A. Sytnik, I. Litvinyuk, Proc. Natl. Acad. Sci. USA 1996, 93, 12959.

[6] a) T. Madrakian, H. Bagheri, A. Afkhami, M. Soleimani, J. Lumin. 2014, 155, 218; b) M. Noronha, R. Santos, E. Paci, H. Santos, A. L. Maçanita, J. Phys. Chem. B 2009, 113, 4466; c) C. Qin, M.-X. Xie, Y. Liu, Biomacromolecule 2007, 8, 2182; d) A. Sułkowska, M. Maciążek-
Jurczyk, B. Bojko, J. Równicka, W. W. Sułkowski, J. Mol. Struct. 2008, 891, 278.

[7] a) B. Sebille, Fundam. Clin. Pharmacol. 1990, 4, 151s; b) I. Vayá, V. Lhiaubet-Vallet, M. C. Jimenez, M. A. Miranda, Chem. Soc. Rev. 2014, $43,4102$.

[8] M. X. Xie, M. Long, Y. Liu, C. Qin, Y. D. Wang, Biochim. Biophys. Acta-Gen. Subj. 2006, 1760 , 1184.

[9] S. Tayyab, M. M. Izzudin, M. Z. Kabir, S. R. Feroz, W. V. Tee, S. B. Mohamad, Z. Alias, J. Photochem. Photobiol. B 2016, 162, 386.

[10] J. R. Lakowicz, 'Principles of Fluorescence Spectroscopy', 3rd ed., Plenum Press, New York, 2006.

[11] W. Y. He, Y. Li, H. Z. Si, Y. M. Dong, F. L. Sheng, X. J. Yao, Z. D. Hu, J. Photochem. Photobiol. A 2006, 182, 158.

[12] a) M. C. Jimenez, M. A. Miranda, R. Tormos, I. Vaya, Photochem. Photobiol. Sci. 2004, 3, 1038; b) V. Lhiaubet-Vallet, Z. Sarabia, F. Bosca, M. A. Miranda, J. Am. Chem. Soc. 2004, 126, 9538; c) M. C. Jimenez, M. A. Miranda, I. Vaya, $J$. Am. Chem. Soc. 2005, 127, 10134; d) I. Vaya, C. J. Bueno, M. C. Jimenez, M. A. Miranda, ChemMedChem 2006, 1, 1015; e) V. LhiaubetVallet, F. Bosca, M. A. Miranda, J. Phys. Chem. B 2007, 111, 423; f) I. Vaya, M. C. Jimenez, M. A. Miranda, J. Phys. Chem. B 2008, 112, 2694; g) R. Perez-Ruiz, C. J. Bueno, M. C. Jimenez, M. A. Miranda, J. Phys. Chem. Lett. 2010, 1, 829; h) I. Vaya, R. Pérez-Ruiz, V. LhiaubetVallet, M. C. Jiménez, M. A. Miranda, Chem. Phys. Lett. 2010, 486, 147; i) R. Perez-Ruiz, R. Alonso, E. Nuin, I. Andreu, M. C. Jimenez, M. A. Miranda, J. Phys. Chem. B 2011, 115, 4460; j) I. Lammers, V. Lhiaubet-Vallet, M. Consuelo Jiménez, F. Ariese, M. A. Miranda, C. Gooijer, Chirality 2012, 24, 840; k) I. Vaya, P. Bonancía, T. Gustavsson, D. Markovitsi, M. C. Jiménez, M. A. Miranda, Phys. Chem. Chem. Phys. 2013, $15,4727$.

[13] a) A. Douhal, M. Sanz, L. Tormo, Proc. Natl. Acad. Sci. USA 2005, 102, 18807; b) M. ElKemary, M. Gil, A. Douhal, J. Med. Chem. 2007, 50, 2896; c) N. Seedher, S. Bhatia, J. Pharm. Biomed. Anal. 2005, 39, 257; d) L. Tormo, J. A. Organero, B. Cohen, C. Martin, L. Santos, A. Douhal, J. Phys. Chem. B 2008, 112, 13641; e) D. Zhong, A. Douhal, A. H. Zewail, Proc. Natl. Acad. Sci. USA 2000, 97, 14056; f) D. Zhong, S. K. Pal, C. Wan, A. H. Zewail, Proc. Natl. Acad Sci. USA 2001, 98, 11873.

[14] a) S. Ercelen, A. S. Klymchenko, Y. Mely, A. P. Demchenko, Int. J. Biol. Macromol. 2005, 35, 231; b) S. S. Sinha, R. K. Mitra, S. K. Pal, J. Phys. Chem. B 2008, 112, 4884; c) N. S. Moyon, S. Mitra, J. Phys. Chem. B 2011, 115 , 10163.

[15] a) J. K. A. Kamal, L. Zhao, A. H. Zewail, Proc. Natl. Acad. Sci. USA 2004, 101, 13411; b) B. Cohen, C. M. Alvarez, N. A. Carmona, J. A. Organero, A. Douhal, J. Phys. Chem. B 2011, 115,7637 ; c) C. Martin, M. Gil, B. Cohen, A. Douhal, Langmuir 2012, 28, 6746; d) C. Martin, B. Cohen, I. Gaamoussi, M. Ijjaali, A. Douhal, J. Phys. Chem. B 2014, 118, 5760.

[16] A. J. Lewis, D. E. Furst, 'Nonsteroidal antiinflammatory drugs: mechanisms and clinical uses', 2nd ed., Marcel Dekker, NewYork, 1994.

[17] A. M. Evans, J. Clin. Pharmacol. 1996, 36, 7S.

[18] T. Gustavsson, D. Markovitsi, I. Vayá, P. Bonancía, M. C. Jiménez, M. A. Miranda, SPIES 2014, $91651 \mathrm{E}$.

[19] T. Wybranowski, M. Cyrankiewicz, B Ziomkowska, S. Kruszewski, Biosystems 2008, 94, 258.

[20] a) J. N. Tian, J. Q. Liu, W. Y. He, Z. D. Hu, X. J. Yao, X. G. Chen, Biomacromolecules 2004, 5, 1956; b) K. Tang, Y. M. Qin, A. H. Lin, X. Hu, G. L. Zou, J. Pharm. Biomed. Anal. 2005, 39 , 404; c) Y. He, Y. W. Wang, L. F. Tang, H. Liu,
W. Chen, Z. L. Zheng, G. L. Zou, J. Fluoresc. 2008, 18, 433; d) H. Liu, W. Bao, H. J. Ding, J. C. Jang, G. L. Zou, J. Phys. Chem. B 2010, 114, 12938; e) H. Vahedian-Movahed, M. R. Saberi, J. Chamani, J. Biomol. Struct. Dyn. 2011, 28 , 483; f) N. Moradi, M. R. Ashrafi-Kooshk, S. Ghobadi, M. Shahlaei, R. Khodarahmi, J. Lumin. 2015, 160, 351; g) M. Shahlaei, B. Rahimi, M. R. Ashrafi-Kooshk, K. Sadrjavadi, R. Khodarahmi, J. Lumin. 2015, 158, 91; h) M. Shahlaei, B. Rahimi, A. Nowroozi, M. R. Ashrafi-Kooshk, K. Sadrjavadi, R. Khodarahmi, Chem.-Biol. Int. 2015, 242, 235.

[21] G. M. Morris, R. Huey, W. Lindstrom, M F. Sanner, R. K. Belew, D. S. Goodsell, A. J. Olson, J. Comp. Chem. 2009, 30, 2785.

[22] a) N. E. Polyakov, V. K. Khan, M. B. Taraban, T. V. Leshina, O. A. Luzina, N. F. Salakhutdinov, G. A. Tolstikov, Org. Biomol. Chem. 2005, 3, 881; b) I. Vayá, I. Andreu, M. C. Jimenez, M. A. Miranda, Photochem. Photobiol. Sci. 2014 13, 224; c) I. Vaya, I. Andreu, V. T. Monje, M. C. Jimenez, M. A. Miranda, Chem. Res. Toxicol. 2016, 29, 40; d) I. Vayá, M. C. Jimenez, M. A Miranda, J. Phys. Chem. B 2007, 111, 9363.

[23] H. Lemmetyinen, N. V. Tkachenko, B. Valeur, J.-i. Hotta, M. Ameloot, N. P. Ernsting, T. Gustavsson, N. Boens, Pure Appl. Chem. 2014, 86, 1969.

[24] a) T. Gustavsson, A. Sharonov, D. Markovitsi, Chem. Phys. Lett. 2002, 351, 195; b) F.-A Miannay, T. Gustavsson, A. Banyasz, D. Markovitsi, J. Phys. Chem. A 2010, 114, 3256.

[25] D. Markovitsi, T. Gustavsson, A. Banyasz, Actualite Chimique 2015, 397-398, 29.

[26] D. Markovitsi, D. Onidas, F. Talbot, S. Marguet, T. Gustavsson, E. Lazzarotto, J. Photochem. Photobiol. A 2006, 183, 1.

[27] M. Montalti, A. Credi, L. Prodi, M. T. Gandolfi, 'Handbook of Photochemistry', CRC Press, Taylor and Francis Group, Boca Raton, FL, 2006.

[28] H. Lemmetyinen, N. Tkachenko, A. Efimov, M. Niemi, J. Porphyrins Phthalocyanines 2009, 13, 1090.

[29] a) S. L. Murov, I. Carmichael, G. L. Hug, 'Handbook of Photochemistry', 2nd ed., Marcel Dekker, 1993; b) J. Pérez-Prieto, F. Bosca, R. Galian, A. Lahoz, L. R. Domingo, M. A. Miranda, J. Org. Chem. 2003, 68, 5104; c) A. Z. Weller, Z. Phys. Chem. 1982, 133, 93.

[30] I. Vayá, T. Gustavsson, D. Markovitsi, M A. Miranda, M. C. Jiménez, J. Photochem. Photobiol. A 2016, 322, 95.

[31] a) Y. Aoki, N. Matsuki, T. Mori, H. Ikeda, Y. Inoue, Org. Lett. 2014, 16, 4888; b) D. M. Guldi, M. Maggini, G. Scorrano, M. Prato, $J$. Am. Chem. Soc. 1997, 119, 974; c) Y. Wang, O. Haze, J. P. Dinnocenzo, S. Farid, J. Org. Chem. 2007, 72, 6970.

[32] T. B. Truong, A. Petit, J. Phys. Chem. 1979, 83, 1300. 